\title{
Mini-access open arch repair
}

\section{Shi A. Kim, Won Kyung Pyo, You Jung Ok, Ho Jin Kim, Joon Bum Kim}

Department of Thoracic and Cardiovascular Surgery, Asan Medical Center, University of Ulsan College of Medicine, Seoul, Republic of Korea

Contributions: (I) Conception and design: SA Kim, JB Kim; (II) Administrative support: JB Kim; (III) Provision of study materials or patients: SA Kim, WK Pyo, YJ Ok, HJ Kim, JB Kim; (IV) Collection and assembly of data: SA Kim; (V) Data analysis and interpretation: SA Kim, WK Pyo, JB Kim; (VI) Manuscript writing: All authors; (VII) Final approval of manuscript: All authors.

Correspondence to: Joon Bum Kim, MD, PhD. Department of Thoracic and Cardiovascular Surgery, Asan Medical Center, University of Ulsan College of Medicine, 88 Olympic-ro 43-gil, Songpa-gu, Seoul 05505, Republic of Korea. Email: jbkim1975@amc.seoul.kr.

Background: The use of minimally invasive approaches is scarce in open aortic arch repair because of its perceived high operative risk and technical difficulty.

Methods: This study enrolled 59 consecutive patients (aged 58.2 \pm 13.2 years) undergoing elective arch replacement either through upper hemi-sternotomy $(n=58)$ or mini-thoracotomy $(n=1)$ between 2015 and 2020. Of these, 44 underwent hemiarch replacement and 15 underwent total arch replacement. Moderate hypothermic circulatory arrest was used for all patients while antegrade cerebral perfusion was selectively used for total arch repair. For more efficient distal aortic anastomosis in limited spaces, inverted graft anastomosis was utilized whenever possible.

Results: Hemi-sternotomy involved upper sternal separation down to the second, third, and fourth intercostal spaces in 1 (1.7\%), 30 (50.8\%), and 27 (45.8\%) patients, respectively. Concomitant cardiac procedures included root replacement in 19 patients $(32.2 \%)$ and aortic valve replacement in 21 patients $(35.6 \%)$. Circulatory arrest, cardiac ischemic, cardiopulmonary bypass, and total procedural times were $8.9 \pm 3.4,91.1 \pm 31.1,114.6 \pm 46.2$, and $250.3 \pm 79.5 \mathrm{~min}$, respectively for total arch repair, and 25.0 \pm 12.1 , $72.3 \pm 16.6,106.0 \pm 16.9$, and $249.1 \pm 41.7 \mathrm{~min}$, respectively for hemiarch repair. Conversion to full-sternotomy was required in 1 patient $(1.7 \%)$ due to bleeding. There was one case of mortality $(1.7 \%)$ attributable to low-cardiac output syndrome following hemiarch repair concomitantly with Bentall procedure. Major complications included requirement for mechanical support in 1 (1.7\%), temporary neurologic deficit in 1 (1.7\%), newly initiated dialysis in 3 (5.1\%), and re-exploration due to bleeding in 2 (3.4\%).

Conclusions: Mini-access open arch repair is technically feasible and achieved excellent early outcomes.

Keywords: Aortic arch surgery; minimally invasive; partial sternotomy

Submitted Nov 08, 2020. Accepted for publication Feb 21, 2021.

doi: $10.21037 /$ jtd-20-3254

View this article at: http://dx.doi.org/10.21037/jtd-20-3254

\section{Introduction}

Since minimally invasive approaches were first introduced to cardiac surgery in the late 1990s, mini-access cardiac surgery has advanced remarkably and has become popular over the last 2 decades (1). Its role in aortic surgery, however, has been limited due to the technical difficulties of the procedures and perceived high operative risks, especially when the surgery involves the aortic arch. Although several studies have described favorable surgical outcomes of mini-access aortic surgery, its safety and efficacy remain controversial because most studies have focused on the replacement of the ascending aorta (2-4). In this regard, we sought to present our surgical strategies and perioperative outcomes of mini-access open arch replacement with the aim of contributing to the body of evidence in this field. We present the following article in accordance with the STROBE reporting checklist (available at http://dx.doi. org/10.21037/jtd-20-3254). 

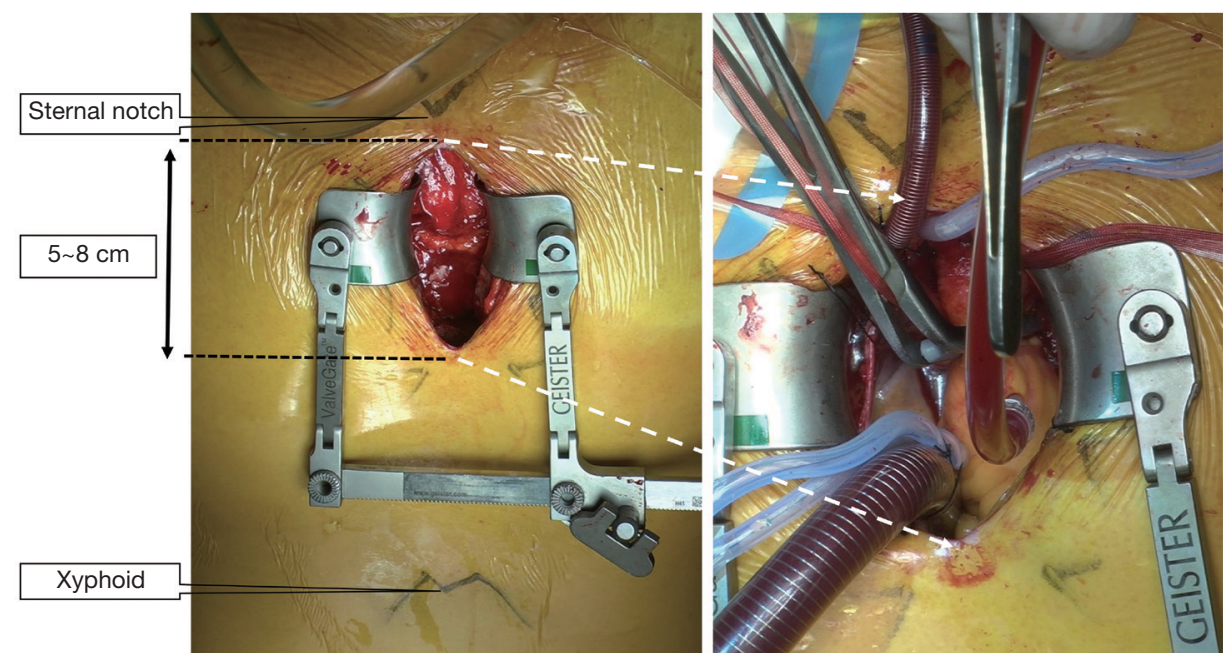

Figure 1 Exposure of the surgical field for total arch replacement via partial sternotomy (down to the third intercostal space).

\section{Methods}

\section{Study population and definitions}

We identified 59 patients who underwent aortic arch repair through minimally invasive approaches to treat aortic diseases involving the arch between August 2015 and April 2020 by a single surgeon (JBK) in the Asan Medical Center, Seoul, Korea. Of 59 patients, 44 underwent hemi-arch replacement (hemiarch group) whereas the remainder $(n=15)$ underwent total arch replacement (total arch group). The study was conducted in accordance with the Declaration of Helsinki (as revised in 2013). The study protocol was approved by the institutional review board of the Asan Medical Center (2020-0739). The requirement for informed consent from individual patients was waived because of the retrospective nature of the present study. The study subjects include 8 patients who were included in our published paper on cerebral perfusion strategy in arch surgery (5).

Hemi-arch replacement was defined as involving the aortic arch beyond the level of the innominate artery but not involving the arch vessels. Total arch replacement was defined as replacing the entire aortic arch by reimplantation of the arch vessels using either individual branch grafting or island anastomosis.

\section{Operative technique}

Either partial sternotomy or anterior right thoracotomy approaches were used. With regard to partial sternotomy, the sternum was partially divided from the manubrium down to the second-fourth intercostal spaces (ICS), with a $5-8 \mathrm{~cm}$ skin incision. In the early study period, upper hemisternotomy down to the fourth intercostal space was the standard approach; however, this was replaced by smaller sternal separation down to the third intercostal space in the later phase. In selected cases with large manubrium, sternotomy down to the second intercostal space only was also used. For hemiarch repairs, upper J-shape hemisternotomy was used and upper-L-shape hemi-sternotomy was used for total arch repairs to better expose the distal arch. In one case, a right anterior thoracotomy incision of $6 \mathrm{~cm}$ was made at the second ICS (Figure 1). The main surgical procedures were performed under moderate hypothermic $\left(25-28^{\circ} \mathrm{C}\right)$ cardiopulmonary bypass, and antegrade administration of del Nido cardioplegic solution (1L) was used for myocardial protection. The surgical procedures and cardiopulmonary strategy employed for hemiarch and total arch replacement were described in our previous study (5). In summary, hemiarch repair was carried out under total circulatory arrest without cerebral perfusion whereas total arch repair was conducted using unilateral antegrade cerebral perfusion.

To facilitate distal anastomosis through a narrow surgical field in this mini-access surgery and for more efficient hemostatic anastomosis using a single layer suture, we utilized an inverted graft technique-a method of invaginating the prosthetic graft into the distal aorta to construct a distal open anastomosis-whenever possible for hemiarch and total arch repairs (Figure 2). For hemiarch repair, the presence of atherosclerosis in the arch that may 
A

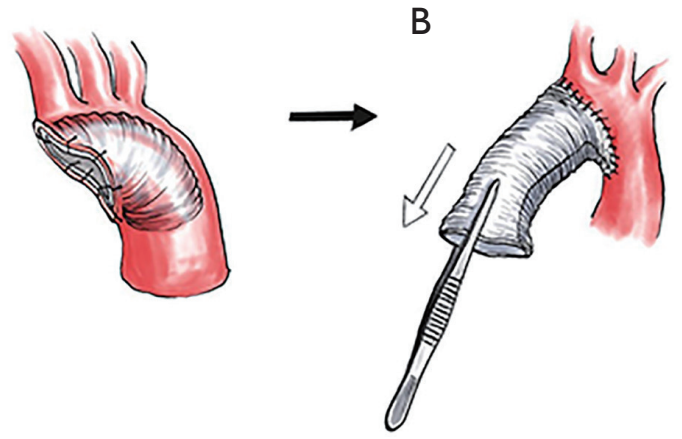

Figure 2 Inverted graft technique: (A) The graft is rolled onto itself in an inside-out fashion and inserted into the proximal descending thoracic aorta. Double layer sutures are placed along the graft in a back-and-forth manner with 3-0 prolene. (B) The invaginated graft is pulled out and ready for anastomosis with the remaining graft.

serve as a potential athero-embolic source was regarded as a contraindication for the use of the inverted grafting technique.

For total arch repair, individual anastomosis using either a 4-branched graft or a separate trifurcate graft was preferred over the island aortic cuff technique for the reconstruction of the arch vessels; however, the latter was preferentially used in individuals without genetic aortopathy when the greater curvature of the arch was free of atherosclerosis or aneurysm. For relatively small distal aorta, where a 4-branch graft cannot be inverted into the graft lumen (i.e., graft size $<28 \mathrm{~mm}$ ), a straight graft was used to construct distal anastomosis using an inverted grafting technique. In these cases, a separate trifurcate graft was used to reconstruct the arch vessels. In cases where a larger 4-branch graft could be used ( $\geq 28 \mathrm{~mm})$, branched portions of the graft were inverted into the distal graft lumen so that the distal anastomosis could be made without interfering with the surgical field by the proximal graft. After distal anastomosis, the inverted portion of the graft was pulled out, and then the arch vessels were subsequently revascularized. For selective cases where either of the above techniques was unavailable, a conventional open distal anastomosis method using a 4-branch arch graft was used (Figure 3).

In selected cases of total arch repair $(\mathrm{n}=5)$, femoral artery cannulation in addition to main cannulation (innominate artery) was made to allow lower body perfusion after distal anastomosis. This procedure was conducted as a replacement of using side-branch of the main aortic graft for lower body perfusion.

For efficient bloodless exposure of the AV, inserting $\mathrm{LV}$ vent cannula through right upper pulmonary vein was a standard approach in cases where concomitant AV/root procedures were combined, while isolated aortic surgeries without concomitant $\mathrm{AV}$ procedures were performed by engaging small vent cannula into the $\mathrm{LV}$ through the $\mathrm{AV}$ from the proximal aortotomy site.

\section{Outcome measures—statistical analyses}

Clinical information was collected through April 2020 by retrieval from institutional electronic medical databases and electronic chart reviews. The outcomes of interest were early mortality and morbidity including low cardiac output syndrome demanding mechanical circulatory support, neurologic deficits, newly initiated dialysis, bleeding requiring surgical intervention, and sternal wound infection requiring re-exploration and prolonged ventilation ( $>48$ hours). Early mortality/morbidity were defined as those occurring within 30 days of surgery or during index hospitalization.

Categorical variables, presented as percentages and frequencies, were compared using Chi-square or Fisher's exact tests. Continuous variables were expressed as the mean \pm standard deviation, and were compared using the Mann-Whitney $U$-test and Student's $t$-test, as appropriate. All reported $\mathrm{P}$ values were two-sided, and $\mathrm{P}$ values less than 0.05 were considered significant. Analyses were performed using R statistical software, version 3.4.00 (R Foundation, Vienna, Austria; httyp://www.R-project.org/).

\section{Results}

\section{Baseline characteristics}

Baseline demographic and clinical profiles are shown in Table 1. Compared with patients in the total arch group, patients in the hemi-arch replacement group were younger $(\mathrm{P}=0.001)$ and had less history of cardiovascular accident $(\mathrm{P}=0.004)$, previous aorta surgery, or intervention $(\mathrm{P}=0.002)$. All four patients with a history of aorta surgery or intervention underwent total arch replacement as follows: thoracic endovascular aortic repair in 2 patients, endovascular aneurysm repair in 1 patient, and distal arch and descending thoracic aorta replacement in 1 patient. Overall, 4 patients were preoperatively diagnosed as 
1.Small distal aorta (graft size $<28 \mathrm{~mm}$ )

$\rightarrow$ Straight graft use

A

B

C
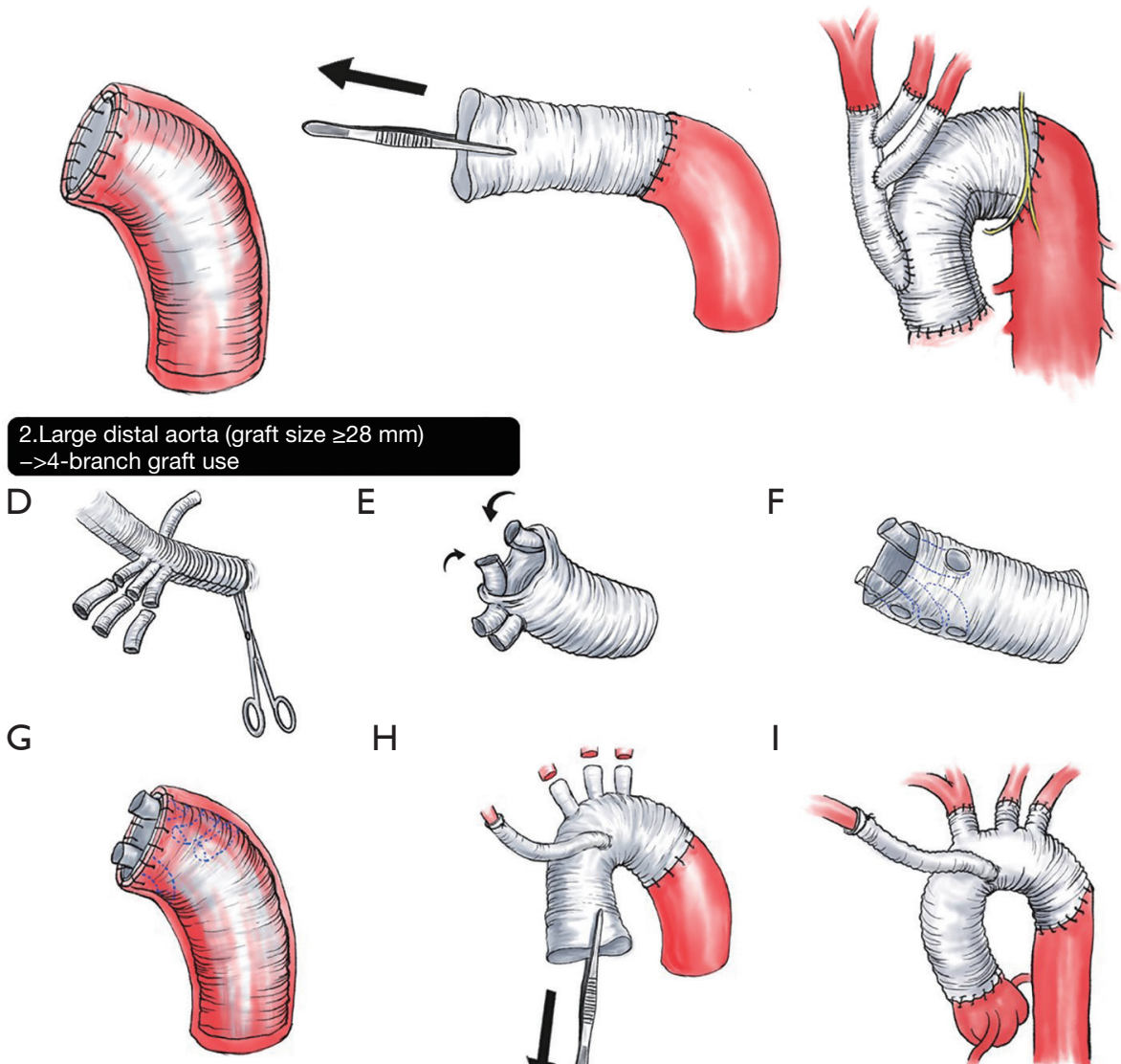

$\mathrm{E}$

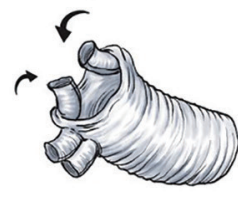

$\mathrm{H}$

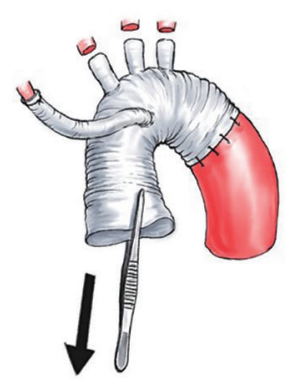

$\mathrm{F}$

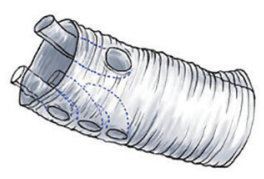

I

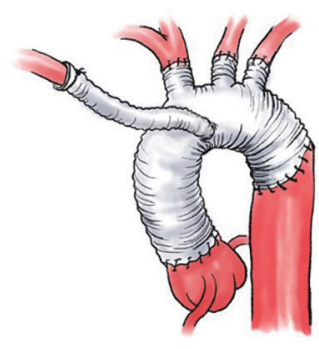

Figure 3 Total arch replacement was performed using the following techniques: (A) For relatively small distal aorta (i.e., graft size <28 $\mathrm{mm}$ ), a straight graft was used to construct distal anastomosis using (B) an inverted grafting technique and (C) a separate trifurcate graft to reconstruct the arch vessels. In cases (D) larger 4-branch graft could be used ( $\geq 28 \mathrm{~mm}$ ), (E) branched portions of the graft were (F) inverted into the distal graft lumen. $(\mathrm{G})$ After distal anastomosis, $(\mathrm{H})$ the inverted portion of the graft was pulled out, and then the arch vessels were subsequently revascularized (I).

Marfan syndrome, and all of them underwent hemiarch replacement along with valve-sparing root replacement due to annuloaortic ectasia.

\section{Operative profiles}

Operative profiles are summarized in Table 2. Regarding the extent of partial sternotomy, an approach down to the third intercostal space was the most common in the overall cohort $(n=30,50.8 \%)$ with a higher proportion in the total arch group $(n=12,80.0 \%)$. Inversely, partial sternotomy down to the fourth intercostal space was more common in the hemi-arch group $(n=24,54.5 \%)$. There was one case of intraoperative full sternotomy conversion to manage massive bleeding in the LV. This case was a 71-year-old woman who underwent aortic valve replacement concomitantly with replacement of the ascending aorta and hemiarch. After decannulation, bleeding from the left lateral side of the heart was detected, which was not fully identifiable by mini-sternotomy. Conversion to full-sternotomy allowed the identification of the bleeder at the antero-lateral side of the LV, and this was controlled by multiple pledgetted 
Table 1 Baseline characteristics

\begin{tabular}{|c|c|c|c|c|}
\hline Characteristics, n (\%) & Overall $(n=59)$ & Hemiarch $(n=44)$ & Total arch $(n=15)$ & $P$ value \\
\hline Male sex & $44(74.6)$ & $32(72.7)$ & $12(80.0)$ & 0.830 \\
\hline \multicolumn{5}{|l|}{ Comorbidities } \\
\hline Hypertension & $28(47.5)$ & $16(36.4)$ & $12(80.0)$ & 0.009 \\
\hline Chronic lung disease & $5(8.5)$ & $5(11.4)$ & 0 & 0.408 \\
\hline Coronary artery disease & $23(39.0)$ & $16(36.4)$ & $7(46.7)$ & 0.689 \\
\hline History of CVA & $23(39.0)$ & $12(27.3)$ & $11(73.3)$ & 0.004 \\
\hline PAOD & $5(8.5)$ & $4(9.1)$ & $1(6.7)$ & $>0.999$ \\
\hline History of cancer & $11(18.6)$ & $8(18.2)$ & $3(20.0)$ & $>0.999$ \\
\hline Marfan disease & $4(6.8)$ & $4(9.1)$ & 0 & 0.539 \\
\hline Previous aorta surgery/intervention & $4(6.8)$ & 0 & $4(26.7)$ & 0.003 \\
\hline Creatinine, mg/dl & $1.0 \pm 0.6$ & $1.0 \pm 0.7$ & $1.0 \pm 0.3$ & 0.894 \\
\hline Hemoglobin, g/dl & $13.2 \pm 1.9$ & $13.4 \pm 2.0$ & $12.4 \pm 1.4$ & 0.063 \\
\hline EuroSCORE II & $2.5 \pm 2.6$ & $2.4 \pm 235$ & $2.7 \pm 2.9$ & 0.706 \\
\hline LV ejection fraction, \% & $60.5 \pm 7.7$ & $59.7 \pm 8.5$ & $62.7 \pm 4.2$ & 0.200 \\
\hline
\end{tabular}

Values are $\mathrm{n}(\%)$ or mean \pm standard deviation, unless otherwise indicated. CVA, cerebrovascular accident; PAOD, peripheral arterial occlusive disease.

sutures. Four hours after the surgery, however, she required second operation due to delayed bleeding, cause of which then being speculated as the $L V$ perforation by the $L V$ vent during the initial operation. At this time, bleeding site was repaired by full-layer pledgetted pure-string sutures of the myocardium, which was reinforced by bovine pericardium patch application on the surface.

For arterial inflow access, the distal ascending aorta was the most commonly selected site in the hemi-arch group $(54.5 \%, \mathrm{n}=21)$, whereas the innominate artery was the most common site in the total arch group. Among the patients undergoing total arch replacement, various techniques for arch vessel anastomosis were employed including the island technique $40.0 \%(\mathrm{n}=6)$, a 4-brach arch graft in $33.3 \%(\mathrm{n}=5)$, and a trifurcate graft in $26.7 \%(n=4)$.

Concomitant cardiac procedures were more commonly undertaken in the hemiarch group, especially aortic root replacement $(43.2 \%$ vs. $0 \%, \mathrm{P}=0.006)$ compared with patients in the total arch group, as well as valve-sparing root replacement in $28.9 \%(13 / 44)$ and the Bentall procedure in $13.3 \%(6 / 44)$ of patients. Aortic cross-clamping time was significantly longer in the hemi-arch group $(90.8 \pm 30.8$ vs. $72.0 \pm 17.2$ minutes, $\mathrm{P}=0.034)$ compared with the total arch group, yet the cardiopulmonary bypass (CPB) time and total operation time were comparable between the groups. 
Table 2 Operative profile

\begin{tabular}{|c|c|c|c|c|}
\hline Characteristics, n (\%) & Overall $(n=59)$ & Hemiarch $(n=44)$ & Total arch $(n=15)$ & $P$ value \\
\hline \multicolumn{5}{|l|}{ Surgical approach } \\
\hline \multicolumn{5}{|l|}{ Partial sternotomy down to } \\
\hline Second ICS & $1(1.6)$ & $1(2.3)$ & 0 & $>0.999$ \\
\hline Third ICS & $30(50.8)$ & $18(40.9)$ & $12(80.0)$ & 0.021 \\
\hline Anterior right thoracotomy & $1(1.7)$ & $1(2.3)$ & 0 & $>0.999$ \\
\hline \multicolumn{5}{|l|}{ Arterial cannulation } \\
\hline Distal ascending aorta & $26(44.1)$ & $21(54.5)$ & $1(6.7)$ & 0.003 \\
\hline Innominate artery & $25(42.4)$ & $15(34.1)$ & $10(66.7)$ & 0.057 \\
\hline \multicolumn{5}{|l|}{ Arch vessel procedure } \\
\hline Individual anastomosis using & & - & & \\
\hline Trifurcate graft & & - & $4(26.7)$ & \\
\hline 4-branched graft & & - & $5(33.3)$ & \\
\hline Island technique & & - & $6(40.0)$ & \\
\hline Elephant trunk & & - & $5(33.3)$ & \\
\hline Inverted graft technique & $20(33.9)$ & $11(25.0)$ & $9(60.0)$ & 0.031 \\
\hline Surgical ablation of AF & $1(1.7)$ & $1(2.3)$ & 0 & 1.000 \\
\hline \multicolumn{5}{|l|}{ Procedural time, minutes } \\
\hline Circulatory arrest time & & $8.9 \pm 3.4$ & $25.0 \pm 12.1$ & $<0.001$ \\
\hline Aortic cross-clamping time & & $91.1 \pm 31.1$ & $72.3 \pm 16.6$ & 0.030 \\
\hline Cardiopulmonary bypass time & & $114.6 \pm 46.2$ & $106.0 \pm 16.9$ & 0.485 \\
\hline Total operation time & & $250.3 \pm 79.5$ & $249.1 \pm 41.7$ & 0.953 \\
\hline Lowest esophageal temperature, ${ }^{\circ} \mathrm{C}$ & & $26.0 \pm 1.2$ & $25.4 \pm 0.7$ & 0.071 \\
\hline
\end{tabular}

Values are $n(\%)$ or mean \pm standard deviation, unless otherwise indicated. AF, atrial fibrillation.

\section{Perioperative outcomes}

There was one case of intraoperative conversion to full-sternotomy $(1.7 \%)$ due to bleeding from the left ventricle-the insult caused by vent cannula. Overall, early mortality occurred in one patient $(1.7 \%)$ who underwent hemi-arch placement. In this 58-year-old man diagnosed as severe bicuspid aortic stenosis combined with 
Table 3 Clinical outcomes

\begin{tabular}{|c|c|c|c|c|}
\hline Adverse clinical outcomes & Overall $(n=59)$ & Hemiarch $(n=44)$ & Total arch $(n=15)$ & $P$ value \\
\hline LCOS requiring MCS & $1(1.7)$ & $1(2.3)$ & - & 0.997 \\
\hline \multicolumn{5}{|l|}{ Neurologic deficit } \\
\hline Permanent neurologic deficit & - & - & - & \\
\hline Newly initiated dialysis & $3(5.1)$ & $2(4.5)$ & $1(6.7)$ & $>0.999$ \\
\hline Reoperation for bleeding & $2(3.4)$ & $2(4.5)$ & - & 0.997 \\
\hline Prolonged ventilation ( $>48$ hours) & $2(3.4)$ & $1(2.3)$ & $1(6.7)$ & 0.997 \\
\hline
\end{tabular}

Values are $\mathrm{n}(\%)$, or median (Q1, Q3) unless otherwise indicated. LCOS, low cardiac output syndrome; MCS, mechanical circulatory support.

bicuspid aortopathy (maximal diameter of $50 \mathrm{~mm}$ ), the initial intention was to perform aortic valve replacement concomitantly with the replacement of the ascending aorta and hemiarch. Bleeding from the aortic root was identified immediately after the release of the aortic clamp, after which a disruption of the thin-walled aortic root was identified. Thereafter, conversion to the Bentall procedure was attempted immediately, but without the need for conversion to full-sternotomy. Despite the successful conversion to the Bentall procedure, the patient failed to be weaned from CPB and suffered from its associated coagulopathy. An extracorporeal membrane oxygenator was applied intraoperatively, and was maintained in the intensive care unit; however, the patient eventually died of multi-organ failure on the third postoperative day despite rigorous efforts to resuscitate this patient. No permanent neurologic deficit occurred in either group; however, 1 patient in the hemi-arch group experienced temporary neurologic deficit presenting as seizure on the day of surgery, but the symptoms subsided after anticonvulsant administration. Brain magnetic resonance imaging revealed a small infarction on the left frontal cortex and multifocal microbleeds, and no further anticonvulsant medication was needed in this patient. Two patients, including the one who died in the perioperative period, underwent ex-exploration for mediastinal bleeding. Other postoperative outcomes are summarized in Table 3.

\section{Discussion}

In concordance with prior studies, the present study demonstrated the favorable perioperative clinical outcomes of mini-access aortic arch $(2,6)$. Even in patients who necessitated concomitant major cardiac procedures (i.e., aortic root replacement), mini-access aortic arch surgery could be undertaken safely and efficaciously, yet its utilization is limited in cased where concomitant mitral valve repair or coronary artery bypass grafting are required.

Several minimally invasive approaches have been proposed for cardiac surgery (7-12) A smaller incision provides a cosmetic advantage as well as clinical benefits such as reduced blood loss, less postoperative pain, and a rapid return to normal life (13-15). In addition, Bonacchi et al., demonstrated that mini-sternotomy improved the recovery of respiratory function (16). These clinical benefits might be enhanced in high-risk patients who are vulnerable to postoperative complications. Despite the clinical advantages and excellent surgical outcomes of mini-access aortic surgery, it should only be used in consultation with a surgeon with technical expertise.

In this study, there was a chronological difference in making the incisions down to $3 \mathrm{rd}$ or 4th intercostal spaces, in that we started with making larger incision (down to 4th ICS) in our earlier experiences when we had not yet performed total arch replacement through mini-incision. After then, we have gained confidence in this surgery, when 
we started making smaller incision (down to 3rd ICS). Around this time and thereafter, mini-access surgery has expanded to total arch replacement. By this reason, most of total arch repairs were conducted by smaller incision (down to 3rd ICS) while roughly latter half of hemiarch repairs were done in this way. J-shape sternal separation was our default approach with the exception of deep total arch repair, in which the distal arch is better exposed by L-shape incision.

We achieved satisfactory perioperative clinical outcomes by using a specific strategy of neuroprotection, CPB management, and standardization of the aforementioned strategies (5). While performing hemi-arch replacement, the hypothermic circulatory arrest technique was adopted, and direct central aortic cannulation was preferred over axillary or femoral cannulation because its safety and efficacy were well established in several previous studies $(11,17,18)$. Regarding the minimization of arrest time and secure hemostasis, distal aortic anastomosis was carried out with a single layer continuous suture, which was reinforced under the restoration of distal perfusion. The early clinical outcomes of our preliminary experiences on mini-access arch repair were satisfactory, and based on these results, this approach may be a useful alternative of conventional sternotomy in the open repair of the arch pathologies $(5,10,12,19)$. However, a limited surgical field is a significant obstacle to overcome in minimally-invasive aortic surgery, especially in patients with a distal arch aneurysm. To enlarge the exposure of the distal arch, we utilized the inverted graft technique for distal anastomosis. Invagination of the graft into the proximal descending thoracic aorta secured the surgical field and a double layer back-and-forth continuous suture along the graft enhanced the hemostatic effect. Comprehensive strategies for surgical approaches, CPB management, neuroprotection, and surgical techniques are mandated for successful minimally-invasive aortic arch surgery in addition to the expertise of the surgeon (10-12).

\section{Limitations}

The present study had several limitations. First, this study has an inherent limitation related to its retrospective nature. Second, the results of this study have limited applicability to patients requiring emergent aortic arch surgery as the minimally invasive approach should only be attempted for patients undergoing elective surgery. Third, this study reported clinical outcomes of aortic arch surgery with or without concomitant cardiac procedures performed by a single surgeon in a high-volume quaternary referral center; thus, the results may not be generalized to other settings. Finally, a larger sample size coupled with a comparison with a conventional sternotomy approach is needed to confirm the findings of the present study.

\section{Conclusions}

Aortic arch surgery can be performed safely and efficaciously with mini-access even for complicated cases requiring concomitant major cardiac procedures. Partial sternotomy or right anterior mini-thoracotomy are reasonable options for patients undergoing non-emergent aortic arch surgery.

\section{Acknowledgments}

The abstract was accepted and was presented at the $34^{\text {th }}$ Annual Meeting of the European Association for Cardio-Thoracic Surgery (EACTS), Barcellona, Spain, October 08-10, 2020. The abstract was accepted and was presented at the $52^{\text {th }}$ Annual Meeting of the Korean Society for Thoracic \& Cardiovascular Surgery, Seoul, Korea, November 05-07, 2020.

Funding: None.

\section{Footnote}

Reporting Checklist: The authors have completed the STROBE reporting checklist. Available at http://dx.doi. org/10.21037/jtd-20-3254

Data Sharing Statement: Available at http://dx.doi. org/10.21037/jtd-20-3254

Peer Review File: Available at http://dx.doi.org/10.21037/jtd20-3254

Conflicts of Interest: All authors have completed the ICMJE uniform disclosure form (available at http://dx.doi. org/10.21037/jtd-20-3254). JBK serves as an unpaid editorial board member of Fournal of Thoracic Disease from Feb 2021 to Jan 2023. The other authors have no conflicts of interest to declare.

Etbical Statement: The authors are accountable for all aspects of the work in ensuring that questions related to the accuracy or integrity of any part of the work are appropriately investigated and resolved. The study was 
conducted in accordance with the Declaration of Helsinki (as revised in 2013). The study protocol was approved by the institutional review board of the Asan Medical Center (2020-0739). The requirement for informed consent from individual patients was waived because of the retrospective nature of the present study.

Open Access Statement: This is an Open Access article distributed in accordance with the Creative Commons Attribution-NonCommercial-NoDerivs 4.0 International License (CC BY-NC-ND 4.0), which permits the noncommercial replication and distribution of the article with the strict proviso that no changes or edits are made and the original work is properly cited (including links to both the formal publication through the relevant DOI and the license). See: https://creativecommons.org/licenses/by-nc-nd/4.0/.

\section{References}

1. Cosgrove DM 3rd, Sabik JF. Minimally invasive approach for aortic valve operations. Ann Thorac Surg 1996;62:596-7.

2. Tabata M, Khalpey Z, Aranki SF, et al. Minimal access surgery of ascending and proximal arch of the aorta: a 9-year experience. Ann Thorac Surg 2007;84:67-72.

3. Svensson LG. Progress in ascending and aortic arch surgery: minimally invasive surgery, blood conservation, and neurological deficit prevention. Ann Thorac Surg 2002;74:S1786-8; discussion S1792-9.

4. Deschka H, Erler S, Machner M, et al. Surgery of the ascending aorta, root remodelling and aortic arch surgery with circulatory arrest through partial upper sternotomy: results of 50 consecutive cases. Eur J Cardiothorac Surg 2013;43:580-4.

5. Park SJ, Kim HJ, Kim JB, et al. Aortic arch repair under moderate hypothermic circulatory arrest with or without antegrade cerebral perfusion based on the extent of repair. J Thorac Dis 2018;10:1875-83.

6. Goebel N, Bonte D, Salehi-Gilani S, et al. Minimally Invasive Access Aortic Arch Surgery. Innovations (Phila) 2017;12:351-5.

7. Doty DB, DiRusso GB, Doty JR. Full-spectrum cardiac surgery through a minimal incision: mini-sternotomy (lower half) technique. Ann Thorac Surg 1998;65:573-7.

Cite this article as: Kim SA, Pyo WK, Ok YJ, Kim HJ, Kim JB. Mini-access open arch repair. J Thorac Dis 2021;13(4):22332241. doi: $10.21037 /$ jtd-20-3254
8. Svensson LG, D'Agostino RS. "J" incision minimal-access valve operations. Ann Thorac Surg 1998;66:1110-2.

9. Kasegawa H, Shimokawa T, Matsushita Y, et al. Rightsided partial sternotomy for minimally invasive valve operation: "open door method". Ann Thorac Surg 1998;65:569-70.

10. Chakos A, Yan TD. Mini-access branch-first total arch replacement and frozen elephant trunk procedure. Ann Cardiothorac Surg 2020;9:236-43.

11. Wu Y, Jiang W, Li D, et al. Surgery of ascending aorta with complex procedures for aortic dissection through upper mini-sternotomy versus conventional sternotomy. J Cardiothorac Surg 2020;15:57.

12. Lentini S, Specchia L, Nicolardi S, et al. Surgery of the Ascending Aorta with or without Combined Procedures through an Upper Ministernotomy: Outcomes of a Series of More Than 100 Patients. Ann Thorac Cardiovasc Surg 2016;22:44-8.

13. Cohn LH, Adams DH, Couper GS, et al. Minimally invasive cardiac valve surgery improves patient satisfaction while reducing costs of cardiac valve replacement and repair. Ann Surg 1997;226:421-6; discussion 427-8.

14. Sun L, Zheng J, Chang Q, et al. Aortic root replacement by ministernotomy: technique and potential benefit. Ann Thorac Surg 2000;70:1958-61.

15. Perrotta S, Lentini S, Rinaldi M, et al. Treatment of ascending aorta disease with Bentall-De Bono operation using a mini-invasive approach. J Cardiovasc Med (Hagerstown) 2008;9:1016-22.

16. Bonacchi M, Prifti E, Giunti G, et al. Does ministernotomy improve postoperative outcome in aortic valve operation? A prospective randomized study. Ann Thorac Surg 2002;73:460-5; discussion 465-6.

17. Frederick JR, Yang E, Trubelja A, et al. Ascending aortic cannulation in acute type a dissection repair. Ann Thorac Surg 2013;95:1808-11.

18. Kamiya H, Kallenbach K, Halmer D, et al. Comparison of ascending aorta versus femoral artery cannulation for acute aortic dissection type A. Circulation 2009;120:S282-6.

19. Kim JB, Chung CH, Moon DH, et al. Total arch repair versus hemiarch repair in the management of acute DeBakey type I aortic dissection. Eur J Cardiothorac Surg 2011;40:881-7. 